\title{
Muscle-fiber transdifferentiation in an experimental model of respiratory chain myopathy
}

Nils Venhoff ${ }^{*}$, Dirk Lebrecht' ${ }^{1}$, Dietmar Pfeifer ${ }^{2}$, Ana C Venhoff ${ }^{1}$, Emmanuel Bissé $^{3}$, Janbernd Kirschner ${ }^{4}$ and Ulrich A Walker ${ }^{1,5^{*}}$

\begin{abstract}
Introduction: Skeletal muscle fiber composition and muscle energetics are not static and change in muscle disease. This study was performed to determine whether a mitochondrial myopathy is associated with adjustments in skeletal muscle fiber-type composition.

Methods: Ten rats were treated with zidovudine, an antiretroviral nucleoside reverse transcriptase inhibitor that induces a myopathy by interfering with mitochondrial functions. Soleus muscles were examined after 21 weeks of treatment. Ten untreated rats served as controls.

Results: Zidovudine induced a myopathy with mitochondrial DNA depletion, abnormalities in mitochondrial ultrastructure, and reduced cytochrome c oxidase activity. Mitochondrial DNA was disproportionally more diminished in type I compared with type || fibers, whereas atrophy predominated in type || fibers. Compared with those of controls, zidovudine-exposed soleus muscles contained an increased proportion (256\%) of type II fibers, whereas neonatal myosin heavy chains remained repressed, indicating fiber-type transformation in the absence of regeneration. Microarray gene-expression analysis confirmed enhanced fast-fiber isoforms, repressed slow-fiber transcripts, and reduced neonatal fiber transcripts in the mitochondrial myopathy. Respiratory chain transcripts were diminished, whereas the enzymes of glycolysis and glycogenolysis were enhanced, indicating a metabolic adjustment from oxidative to glycolytic capacities. A coordinated regulation was found of transcription factors known to orchestrate type II fiber formation (upregulation of MyoD, Six1, Six2, Eya1, and Sox6, and downregulation of myogenin and ERR $\gamma$ ).
\end{abstract}

Conclusions: The type I to type II fiber transformation in mitochondrial myopathy implicates mitochondrial function as a new regulator of skeletal muscle fiber type.

\section{Introduction}

Low muscle endurance and fatigue are frequent symptoms of patients with diseases that limit the oxygen supply of muscles by its capillaries or muscular oxygen use by its mitochondria. Muscle capillaries are lost in dermatomyositis [1], systemic sclerosis [2-4], and chronic obstructive pulmonary disease (COPD) [5], and qualitative or quantitative defects of respiratory chain components are found in the mitochondrial myopathies [6,7]. Ultrastructural

\footnotetext{
* Correspondence: nils.venhoff@uniklinik-freiburg.de; ulrich.walker@fps-basel. ch

'Department of Rheumatology \& Clinical Immunology, University Medical Center Freiburg, Hugstetter Str. 55, Freiburg, 79104, Germany

Full list of author information is available at the end of the article
}

changes in mitochondria and respiratory-chain dysfunction can also be induced by medications (statins [8], zidovudine, and other antiretroviral nucleoside analogues [9], and potentially, alcohol [10]). The physiological explanations for muscle fatigue and the adjustments of muscle metabolism to such respiratory compromise have, however, been only poorly addressed.

In humans, most skeletal muscles are equipped with more than one fiber type to accommodate a wide range of forces, kinetics, and endurance. Muscles specialized for maintaining postural tone have a high proportion of fibers that contract slowly (type 1 fibers), whereas muscles specialized for rapid movements contain a high proportion of

\section{Biomed Central}

(c) 2012 Venhoff et al.; licensee BioMed Central Ltd. This is an open access article distributed under the terms of the Creative Commons Attribution License (http://creativecommons.org/licenses/by/2.0), which permits unrestricted use, distribution, and reproduction in any medium, provided the original work is properly cited. 
fast-twitch (type 2) fibers. To account for fiber-type diversity, virtually every contractile protein of muscle fibers exists in different isoforms. Muscle-fiber types have also developed fine-tuned systems of energy delivery, which result in diverse metabolic profiles and oxygen requirements. Fiber types are, however, not static, as endurance training, weight loading, or hormonal factors can promote fiber-type transformation, even in adult muscles, by means of a coordinated antithetic regulation of fast and slow gene programs [11-13]. No study has investigated skeletal muscle fiber-type adjustments in response to a primary defect of the mitochondrial respiratory chain.

We therefore investigated how skeletal muscles adjust to mitochondrial dysfunction and whether they can alter their fiber-type composition. In this study, we modeled a mitochondrial myopathy by feeding rats with zidovudine, a nucleoside-analogue reverse transcriptase inhibitor that impairs with the replication of mitochondrial DNA and interferes with mitochondrial function through a variety of mechanisms, including competition with the normal nucleotide triphosphates for incorporation into replicating mtDNA chains, impairment of chain elongation, and excision-repair steps (extensively reviewed elsewhere) [14]. On a global basis, zidovudine is widely used in the treatment of human immunodeficiency virus (HIV) infections and can also cause a myopathy in humans $[9,15]$. Our experiments are the first to describe the ability of skeletal muscle to change fiber-type composition by downregulating the proportion of slow fibers and upregulating fast fibers in response to mitochondrial dysfunction. The changes in fiber-type composition are accompanied by metabolic adjustments from oxidative to more glycolytic capacities.

\section{Materials and methods}

\section{Animals}

Male Wistar rats were purchased at Charles River (Sulzfeld, Germany), were fed a normal rat chow (SSniff R/ M-H; Spezialdiäten, Soest, Germany) ad libitum, and were housed in a normal night-day rhythm under standard conditions of temperature and humidity. At 7 weeks of age, 10 rats received zidovudine (kindly provided by GlaxoSmithKline, Munich, Germany) in the drinking water $(100 \mathrm{mg} / \mathrm{kg} / \mathrm{d})$. This daily dose of zidovudine corresponds to the human dosage adjusted for body area and the higher metabolic and drug-disposal rate of rodents and was calculated on the basis of a daily liquid consumption of $20 \mathrm{ml}[16,17]$. Control rats $(n=10)$ did not receive any zidovudine.

Observations for fluid consumption, clinical signs, and mortality were carried out daily; body weights were recorded weekly. All rats were killed by cervical dislocation at age 28 weeks, immediately before organ collection and postmortem examination. Soleus muscle was snap frozen and cryopreserved in liquid nitrogen until subsequent analysis. Muscle aliquots were fixed in glutaraldehyde (3\%) for subsequent electron microscopy. Serum was collected by puncture of the Venae saphenae laterales [18] before cervical dislocation in anesthesia with isoflurane (Abbott, Wiesbaden, Germany). All animal work was performed after animal welfare board approval (Regierungspräsidium Freiburg; Department 3, Nr. 35/9185/.81/G-07/67) and conformed to institutional guidelines as well as to the $\mathrm{NIH}$ policy [19].

\section{Histopathology and mitochondrial ultrastructure}

Soleus muscle-fiber diameters were morphometrically quantified in all rats on three randomly selected $0.09-\mathrm{mm}^{2}$ areas of $8 \mu \mathrm{m}$ thick, hematoxylin and eosin-stained sections, by using an automated image-analysis and processing software (Leica QWin Standard v2.7; Leica Microsystems, Imaging Solutions, Cambridge, UK). The histochemical assay for myofibrillar ATPase activity (pH 4.35 or 10.5) was used to distinguish and morphometrically count fast and slow muscle fibers [20]. On 4- $\mu \mathrm{m}$ cryostat muscle transverse sections, succinate dehydrogenase (SDH) and cytochrome $c$-oxidase (COX) histochemistry was performed [21]. The evaluating person was blinded to the group status of all animals. Two randomly selected soleus muscle samples from each group were examined with electron microscopy, as described [22].

\section{Myosin heavy-chain immunohistochemistry}

Fiber-type analyses were confirmed in muscle cryosections $(8 \mu \mathrm{m})$ incubated overnight with 1:100 diluted antibodies against fast or slow myosin heavy-chain isoforms (clones WB-MHCF and WB-MHCS; Novocastra, Newcastle, UK). To determine signs of regenerating fibers, we used anti-neonatal myosin heavy-chain antibody (clone WB-MHCn; Novocastra). An Alexa-fluor 488 conjugated secondary antibody (IgG anti-mouse; Novacastra) was used. In images obtained from immunohistochemistry, muscle fiber-type composition was also quantified by using automated image analysis (Leica QWin Standard v2.7; Leica Microsystems). The evaluating person was blinded to the group status of the animals.

\section{Respiratory-chain enzyme activities}

Histochemical COX and SDH staining is difficult to quantify reliably. We therefore measured the activities of $\mathrm{COX}, \mathrm{SDH}$, and nicotinamide adenine dinucleotide hydrogen dehydrogenase (NADH-DH) in freshly prepared soleus muscle extracts with spectrophotometric assays, as described [23]. NADH-DH and COX are the multisubunit complexes I and IV of the mitochondrial respiratory chain and are encoded partly by nuclear DNA (nDNA) and partly by mtDNA, whereas SDH is a respiratory chain component (complex II), which is encoded entirely by nDNA. 


\section{Serum parameters}

Serum concentrations of creatinine kinase, resting lactate, glucose, aspartate aminotransferase, alanine aminotransferase, and creatinine levels were determined photometrically by using a Roche/Hitachi 917/Modular P analyzer (Mannheim, Germany), according to the manufacturer's instructions.

\section{Single-fiber mtDNA copy numbers}

In each animal, three fast and three slow fibers were picked with a microcapillary under an inverted microscope from a 14- $\mu$ m-thick, ATPase activity ( $\mathrm{pH} 10.5)$ typed, transverse soleus muscle section [24]. Total DNA from single fibers was released with $5 \mu$ l of a solution containing $200 \mathrm{mM} \mathrm{KOH}$ and $50 \mathrm{~m} M$ dithiothreitol (incubated for 1 hour at $65^{\circ} \mathrm{C}$ ), followed by a neutralizing buffer $(5 \mu \mathrm{l})$ containing $900 \mathrm{~m} M$ Tris- $\mathrm{HCl}, \mathrm{pH} 8.3$, and $200 \mathrm{mM} \mathrm{HCl} \mathrm{[24].} \mathrm{MtDNA} \mathrm{and} \mathrm{nDNA} \mathrm{copy} \mathrm{numbers}$ were quantified from $2 \mu \mathrm{l}$ of the solute by quantitative PCR, as described [25]. Amplifications of mitochondrial and nuclear products were performed in triplicate. Absolute mtDNA and nDNA copy numbers were calculated by using serial dilutions of plasmids with known copy numbers.

\section{Microarray analysis}

RNA was extracted from eight randomly selected frozen muscles from each group with the Uneasy Kit (Qiagen, Hilden, Germany). Quantity and integrity of the RNA were verified by using RNA 6000 nano chips (2100 Bioanalyzer; Agilent, Palo Alto, CA, USA). RNA samples (500 ng) with an RNA integrity number of greater than 9 were further processed with the GeneChip Whole Transcript Sense Target Labelling Assay from Affymetrix (Santa Clara, CA, USA) according to the manufacturer's instructions.

Arrays were scanned with the Affymetrix GeneChip Scanner $30007 \mathrm{G}$, and raw data were imported into the Refiner module of Genedata Expressionist software (Martinsried, Germany, version 5.3.5), in which quantile normalization and probe summarization was performed by using its Refiner condensing algorithm [26]. The microarray data were uploaded (ArrayExpress accession number: E-MEXP-3642) in the ArrayExpress Archive [27].

\section{Statistics}

The Kolmogorov-Smirnov test was used to analyze for normal distribution. Groups were then compared with ANOVA, Mann-Whitney, unpaired $t$ test, or Wilcoxon analysis, as appropriate. Skewed data are provided as median plus interquartile ranges (IQRs), and normally distributed data, as group means and standard deviation (SD). Correlations were computed as nonlinear exponential regressions. All graphics and calculations were performed by using the Sigma Plot 2000, version 8.0 (SPSS, Inc.) and the Sigma Stat, version 3.1 (Jandel Inc.) packages.

To identify differentially expressed genes between the groups in microarray analysis, the unpaired Bayes $T$ test (CyberT) [26] with the Bayes confidence estimate value set to 24 and a window size of 101 genes, as well as $100 \%$ valid values in each group, was performed with the Analyst module of Expressionist. To estimate the false-discovery rate, the Benjamini-Hochberg $q$ value was calculated in a sequential Bonferroni-type procedure [28]. We then used the "N-fold regulation" activity of Analyst to calculate the median ratio between the experimental groups. Only genes from the categories "main" and "unmapped" (see Affymetrix transcript annotation RaGene-1_0-st-v1.na30.1. rn4.transcript) were included, thereby omitting control probes or genes with uncertain annotation. The falsediscovery rate, which estimates the number of false positives within a list of significant genes, was chosen as $10 \%$.

\section{Results}

\section{Zidovudine induces a respiratory-chain myopathy}

The daily fluid consumption and body weight of the rats was unaffected by zidovudine (data not shown). The autopsy did not reveal macroscopic organ anomalies. Soleus muscle fiber diameters were decreased in the zidovudine group (Figure 1, Table 1). After 28 weeks,
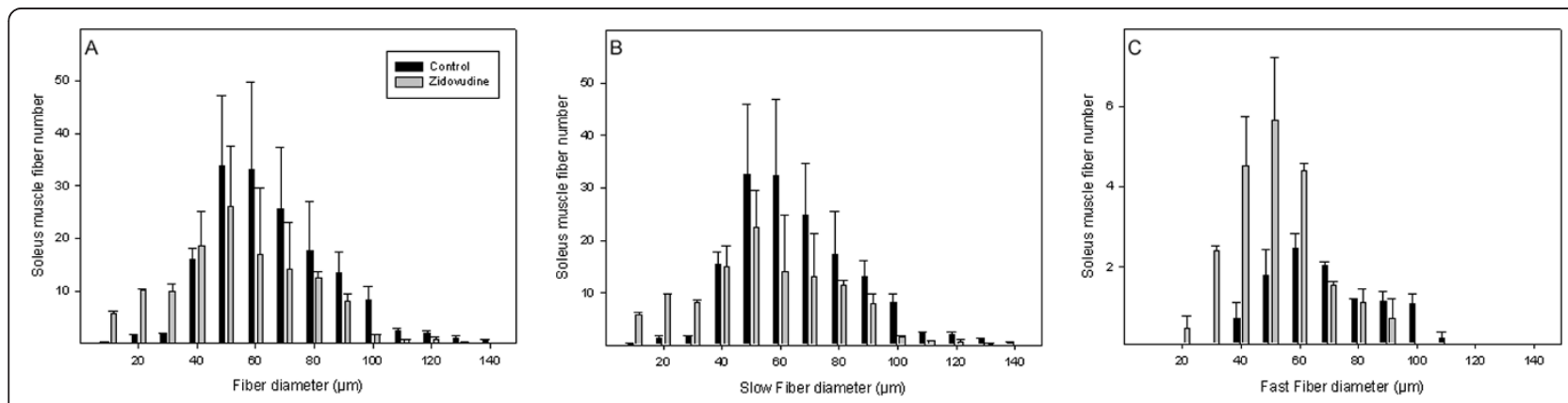

Figure 1 Average numbers of soleus muscle fiber diameters (A). Fiber thinning in type I (B) and in type II fibers (C). Values represent group means $( \pm S D)$. 
Table 1 Effects of zidovudine on soleus muscle histology, serum parameters, mtDNA, mitochondrial function, and muscle fiber-type characteristics

\begin{tabular}{|c|c|c|c|c|c|c|}
\hline Complete soleus muscle & \multicolumn{2}{|c|}{ Control $(n=10)$} & \multicolumn{2}{|c|}{ Zidovudine $(n=10)$} & \multicolumn{2}{|c|}{$P$ value } \\
\hline Soleus muscle fiber diameter (median $\mu \mathrm{m}$ ) & \multicolumn{2}{|c|}{58.3 (IQR 54.2, 62.3) } & \multicolumn{2}{|c|}{41.1 (IQR 37.5, 47.1) } & \multicolumn{2}{|l|}{$<0.001$} \\
\hline Serum creatine kinase $(U / L)$ & \multicolumn{2}{|l|}{$724 \pm 480$} & \multicolumn{2}{|l|}{$548 \pm 257$} & \multicolumn{2}{|l|}{0.3} \\
\hline Serum creatinine (mg/L) & \multicolumn{2}{|l|}{$3.6 \pm 0.34$} & \multicolumn{2}{|l|}{$3.1 \pm 0.11$} & \multicolumn{2}{|l|}{$<0.001$} \\
\hline Serum glucose (mg/L) & \multicolumn{2}{|l|}{$1,760 \pm 100$} & \multicolumn{2}{|l|}{$1,680 \pm 220$} & \multicolumn{2}{|l|}{0.055} \\
\hline Serum resting lactate $(\mathrm{m} M)$ & \multicolumn{2}{|l|}{$4.5 \pm 1.8$} & \multicolumn{2}{|l|}{$5.0 \pm 1.6$} & \multicolumn{2}{|l|}{0.9} \\
\hline Serum alanine aminotransferase (U/L) & \multicolumn{2}{|l|}{$66 \pm 8$} & \multicolumn{2}{|l|}{$56 \pm 7$} & \multicolumn{2}{|l|}{0.1} \\
\hline Serum aspartate aminotransferase $(\mathrm{U} / \mathrm{L})$ & \multicolumn{2}{|l|}{$125 \pm 35$} & \multicolumn{2}{|l|}{$101 \pm 13$} & \multicolumn{2}{|l|}{0.09} \\
\hline mtDNA copy number (mean copies/myonucleus) & \multicolumn{2}{|l|}{$559 \pm 46$} & \multicolumn{2}{|l|}{$399 \pm 78$} & \multicolumn{2}{|l|}{$<0.001$} \\
\hline NADH DH ( $\mu$ moles min $^{-1} \mathrm{~g}$ muscle protein ${ }^{-1}$ ) & \multicolumn{2}{|l|}{$684 \pm 254$} & \multicolumn{2}{|l|}{$474 \pm 236$} & \multicolumn{2}{|l|}{0.042} \\
\hline $\operatorname{COX}\left(\mu\right.$ moles $\min ^{-1} \mathrm{~g}$ muscle protein $\left.{ }^{-1}\right)$ & \multicolumn{2}{|l|}{$28 \pm 8$} & \multicolumn{2}{|l|}{$18 \pm 9$} & \multicolumn{2}{|l|}{0.026} \\
\hline $\mathrm{SDH}$ ( $\mu$ moles $\mathrm{min}^{-1} \mathrm{~g}$ muscle protein ${ }^{-1}$ ) & \multicolumn{2}{|l|}{$36 \pm 6$} & \multicolumn{2}{|l|}{$35 \pm 10$} & \multicolumn{2}{|l|}{0.7} \\
\hline NADH DH/SDH-ratio (\% of control mean) & $100 \pm 28$ & & $72 \pm 20$ & & 0.027 & \\
\hline COX/SDH-ratio (\% of control mean) & $100 \pm 27$ & & $67 \pm 26$ & & 0.016 & \\
\hline Single-fiber soleus muscle & Control & & Zidovudine & & $p$-value & \\
\hline Fiber type & Fast & Slow & Fast & Slow & Fast & Slow \\
\hline Fiber-type proportion (\% of all fibers) & $5.2 \pm 3.0$ & $94.8 \pm 3.0$ & $13.3 \pm 5.1$ & $86.7 \pm 5.1$ & $<0.001$ & $<0.001$ \\
\hline Fiber diameter (mean $\mu \mathrm{m})$ & $54.8 \pm 5.8$ & $65.5 \pm 10.6$ & $37.3 \pm 9.3$ & $51.8 \pm 6.2$ & $<0.001$ & 0.006 \\
\hline mtDNA copy number (mean copies/myonucleus) & $617 \pm 91$ & $683 \pm 94$ & $384 \pm 91$ & $206 \pm 97$ & $<0.001$ & $<0.001$ \\
\hline
\end{tabular}

Values represent group means $( \pm \mathrm{SD})$; soleus muscle-fiber diameter values are given as median with interquartile range.

groups did not differ in serum levels of creatinine kinase, resting lactate, and glucose. Serum creatinine levels, however, were lower in rats treated with zidovudine, indicating reduced muscle mass $(P=0.001)$ compared with untreated rats. Electron microscopy revealed a focal disarray of the myofibrillar lattice in the zidovudine group (Figure 2). The crystal architecture was lost in a substantial proportion of the organelles and contained deposits of electron-dense material. Mean mtDNA copy numbers were decreased by $29 \%(P<0.001)$ in zidovudine-treated rats compared with control animals (Table 1 ). Histochemical COX/SDH staining showed a uniformly downregulated respiratory-chain activity and no clear fiber type-specific pattern. NADH-DH and COX activities in the soleus muscle were depressed in the zidovudine group $(P=0.042$ and $P=0.026$, respectively; Table 1$)$. In contrast, the activity of SDH was unaffected $(P=0.7$; Table 1). These data indicate that zidovudine induced a metabolic myopathy with depleted mtDNA copies and a specific downregulation of mtDNA-encoded respiratory chain activities and consecutive fiber atrophy.

Type II fibers are enhanced in mitochondrial myopathy Muscle morphometry revealed that the fiber diameter was reduced in both fast and slow fibers of zidovudine-treated rats (Table 1), leading to an increased fiber number per microscopic area $\left(151 \pm 20\right.$ fibers $/ \mu \mathrm{m}^{2}$ in rats without versus $194 \pm 26$ fibers $/ \mu \mathrm{m}^{2}$ in rats with zidovudine; $P=0.002)$. Fast fibers, however, had a disproportionate degree of atrophy (32\% reduction of mean fiber diameter; $P<0.001)$ compared with slow fibers $(21 \%$ reduction; $P=0.006)$.

In control muscle, slow fibers and fast fibers contained similar numbers of mtDNA copies $(P=0.15)$. In zidovudine-treated rats, however, slow fibers had fewer mtDNA copies than did fast fibers $(P=0.002)$. Zidovudine treatment also induced a greater proportion of mtDNA depletion in slow fibers than in fast fibers $(70 \%$ versus $38 \% ; P<0.001$; Table 1 ).

As expected, histomorphometry in ATPase-typed soleus muscle of control rats demonstrated the vast predominance of type 1 slow oxidative fibers $(94.8 \% \pm$ $3.0 \%)$ relative to fast glycolytic fibers. In contrast, zidovudine-treated animals, soleus muscles contained a high proportion of fast fibers (256\% increase compared with controls; $P<0.001$ ), whereas slow fibers were diminished (Table 1). Fiber-type grouping was not evident. The upregulation of fast fibers in soleus muscle exposed to zidovudine was confirmed with antibodies specific for fast and slow myosin heavy chain (Figure 3). Immunofluorescence studies of zidovudine-treated soleus muscles were carried out with an antibody directed against neonatal MHC. None of the soleus muscle fibers expressed the neonatal myosin heavy-chain isoform, indicating the absence of fiber regeneration (not shown). Furthermore, we did not observe signs of muscle denervation in terms of fiber-type grouping or upregulated neonatal myosin heavy chains (data not shown). 

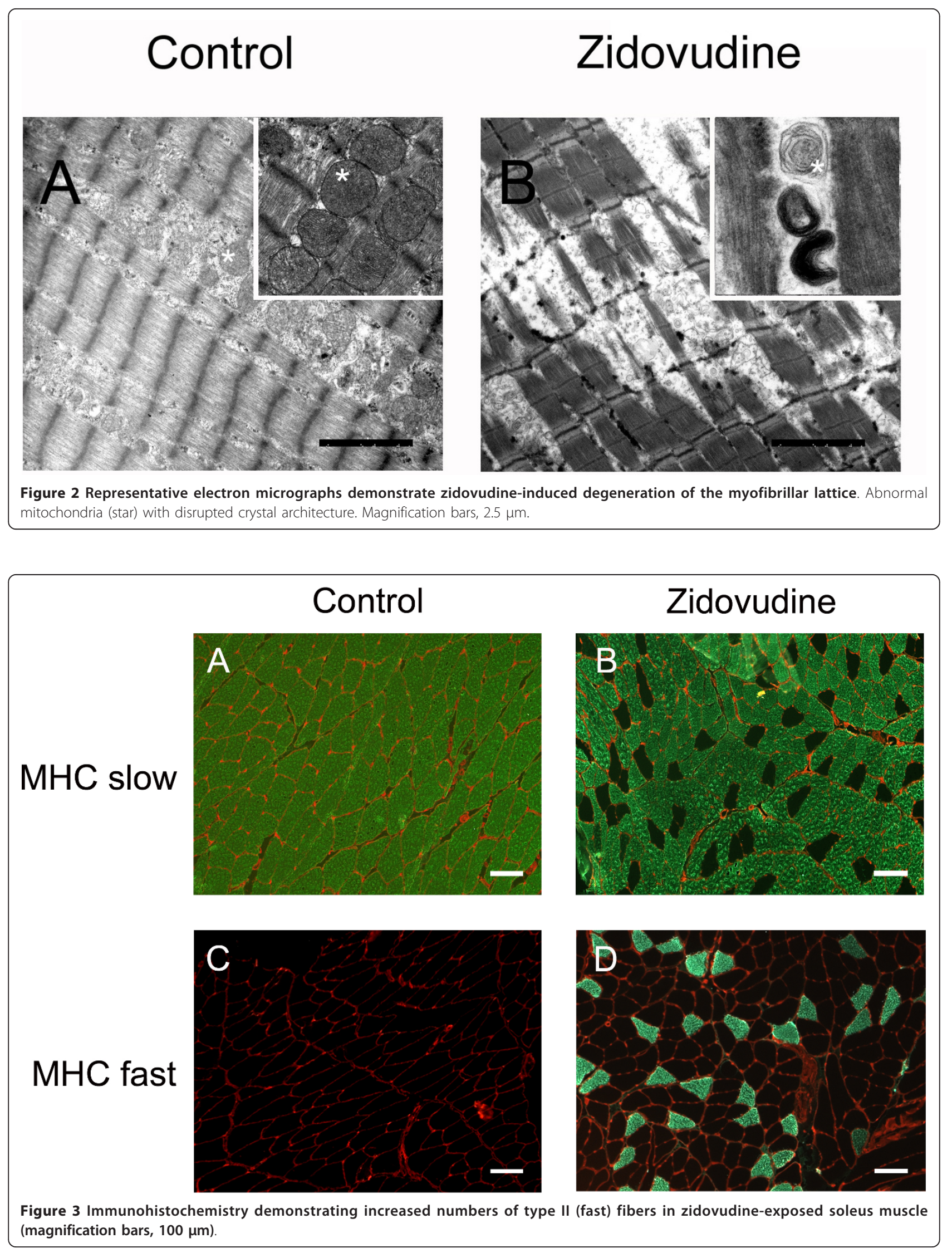
Enhanced fast-fiber and repressed slow-fiber transcripts Microarray gene expression analysis identified 1,411 genes and 43 pathways to be significantly regulated $(P<$ 0.01 ) in zidovudine-treated animals relative to control. In zidovudine-exposed rat soleus muscle, fast-fiber transcripts were significantly enhanced, and slow-fiber transcripts, repressed (Figure 4). Consistent with the absence of fiber regeneration in the immunofluorescence studies, embryonal and neonatal myosin heavy-chain transcripts were downregulated [29].

Investigating metabolic adjustments, we found nucleusand mtDNA-encoded respiratory chain subunits to be coordinately downregulated in zidovudine myopathy, although many changes were not statistically significant. The transcription of the rate-limiting enzymes of glycolysis and glycogenolysis was enhanced (Table 2) and the mitochondrial carnitine shuttle (carnitine palmitoyltransferase, $C P T 1 b$ ), and $\beta$-oxidation (3-hydroxy-acyl-CoA dehydrogenase) downregulated.

\section{Regulation of fiber composition}

To elicit regulatory mechanisms of fiber transformation, we focused on transcription factors involved in muscle differentiation. In zidovudine-treated muscle, MyoD, which is expressed mainly in type II fibers [30], was upregulated $(+4.41$-fold; $P=1.01 \mathrm{E}-16)$, and the myogenic

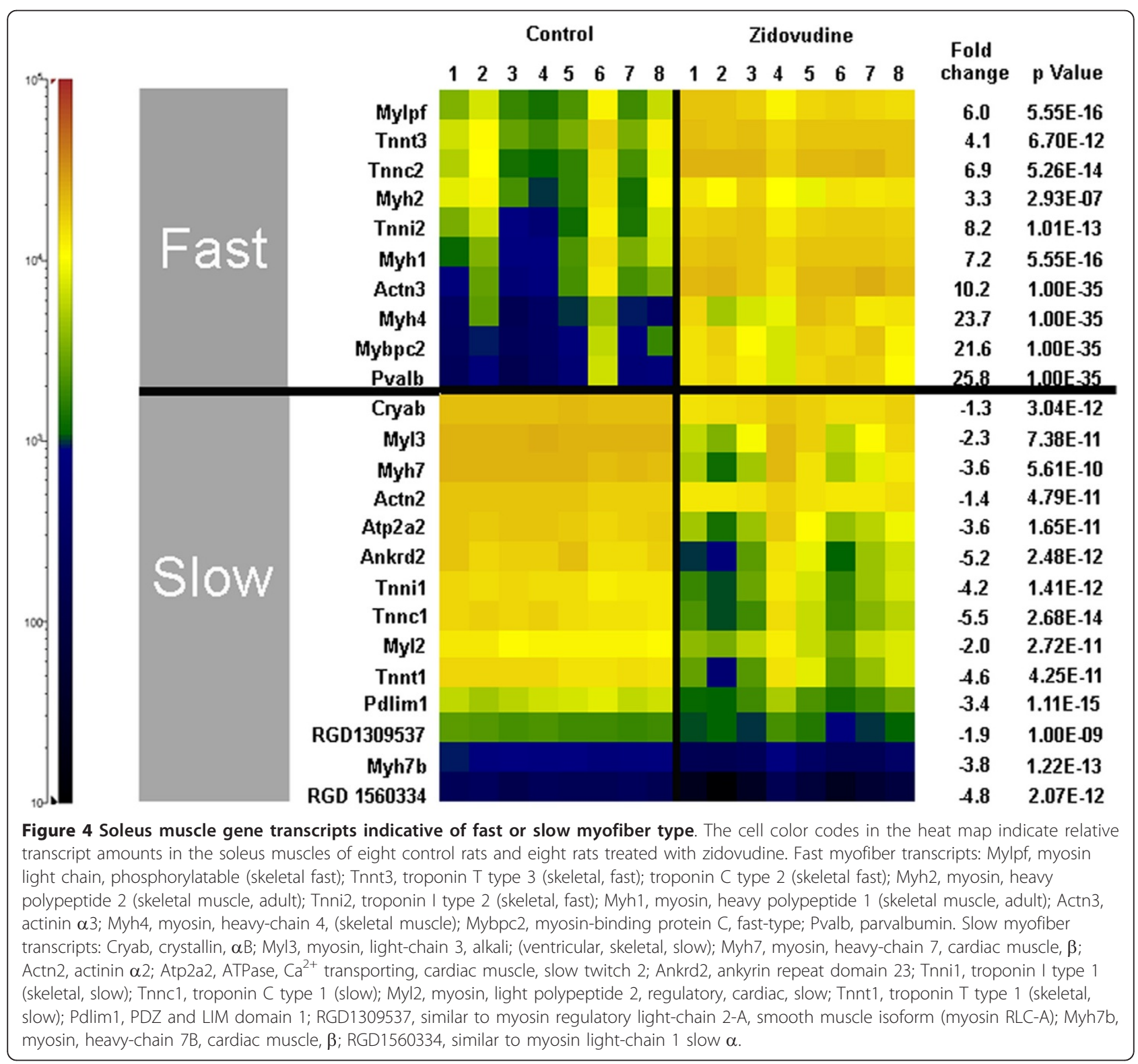


Table 2 Microarray gene expression of soleus muscle-fiber energy metabolism and gene products involved in fibertype regulation

\begin{tabular}{|c|c|c|c|}
\hline Gene description (gene symbol) & FC & $P$ value & $q$ value \\
\hline \multicolumn{4}{|l|}{ Respiratory chain complex I } \\
\hline NADH dehydrogenase (ubiquinone) $1 \alpha$ subcomplex, 4 (Ndufa4) & -1.1 & 0.007 & 0.999 \\
\hline NADH dehydrogenase (ubiquinone) $1 \alpha / \beta$ subcomplex, 1 (Ndufab1) & 1.0 & 0.507 & 0.999 \\
\hline NADH dehydrogenase (ubiquinone) $1 \alpha$ subcomplex, assembly factor 1 (Ndufaf1) & -1.1 & 0.110 & 0.999 \\
\hline NADH dehydrogenase (ubiquinone) $1 \beta$ subcomplex 3 (Ndufb3) & -1.0 & 0.772 & 0.999 \\
\hline NADH dehydrogenase (ubiquinone) $1 \beta$ subcomplex, 5 (Ndufb5) & -1.1 & 0.036 & 0.999 \\
\hline NADH dehydrogenase (ubiquinone) $1 \beta$ subcomplex, 9 (Ndufb9) & -1.0 & 0.286 & 0.999 \\
\hline NADH dehydrogenase (ubiquinone) 1, subcomplex unknown, 1 (Ndufc1) & -1.1 & 0.167 & 0.999 \\
\hline NADH dehydrogenase (ubiquinone) Fe-S protein 1 (Ndufs1) & -1.1 & 0.045 & 0.999 \\
\hline NADH dehydrogenase subunit 1 (mt-ND1) & -1.1 & 0.267 & 0.999 \\
\hline NADH dehydrogenase subunit 2 (mt-ND2) & -1.1 & 0.087 & 0.999 \\
\hline NADH dehydrogenase subunit 4L (mt-ND4l) & -1.1 & 0.004 & 0.999 \\
\hline NADH dehydrogenase subunit 5 (mt-ND5) & -1.1 & 0.002 & 0.999 \\
\hline NADH dehydrogenase subunit 6 (mt-ND6) & -1.2 & 0.364 & 0.999 \\
\hline \multicolumn{4}{|l|}{ Respiratory chain complex II } \\
\hline Succinate dehydrogenase complex, subunit B, iron sulfur (Ip) (Sdhb) & -1.4 & 0.00001 & 0.139 \\
\hline \multicolumn{4}{|l|}{ Respiratory chain complex III } \\
\hline Ubiquinol-cytochrome $\mathrm{c}$ reductase binding protein (Uqcrb) & -1.1 & 0.003 & 0.999 \\
\hline Ubiquinol-cytochrome c reductase core protein I (Uqcrc1) & -1.0 & 0.677 & 0.999 \\
\hline Ubiquinol cytochrome $\mathrm{c}$ reductase core protein 2 (Uqcrc2) & -1.1 & 0.039 & 0.999 \\
\hline \multicolumn{4}{|l|}{ Respiratory chain complex IV } \\
\hline Cytochrome c oxidase, subunit Va (Cox5a) & -1.1 & 0.205 & 0.999 \\
\hline Cytochrome c oxidase subunit Vb (Cox5b) & -1.0 & 0.442 & 0.999 \\
\hline Cytochrome c oxidase, subunit Vla, polypeptide 2 (Cox6a2) & -1.1 & 0.096 & 0.999 \\
\hline Cytochrome $c$ oxidase, subunit VIc (Cox6c) & -1.1 & 0.144 & 0.999 \\
\hline Cytochrome $c$ oxidase subunit VIlb (Cox7b) & 1.0 & 0.501 & 0.999 \\
\hline Cytochrome c oxidase, subunit VIla 2 (Cox7a2) & -1.1 & 0.006 & 0.999 \\
\hline Cytochrome c oxidase subunit VIla polypeptide 2 like (Cox7a2l) & -1.2 & 0.002 & 0.999 \\
\hline Cytochrome $\mathrm{c}$ oxidase subunit I (mt-Co1) & -1.1 & 0.000 & 0.999 \\
\hline Cytochrome c oxidase subunit II (mt-Co2) & -1.1 & 0.028 & 0.999 \\
\hline \multicolumn{4}{|l|}{ Respiratory chain complex $\mathrm{V}$} \\
\hline ATP synthase, F1 complex, $\gamma$ polypeptide 1 (Atp5c1) & 1.0 & 0.807 & 0.999 \\
\hline ATP synthase, F0 complex, subunit C3 (subunit 9) (Atp5g3) & -1.1 & 0.080 & 0.999 \\
\hline ATP synthase GA binding protein transcription factor, $\alpha$ subunit: (Gabpa: Atp5j) & -1.2 & 0.005 & 0.999 \\
\hline ATP synthase, F0 complex, subunit F2 (Atp5j2) & 1.0 & 0.548 & 0.999 \\
\hline ATP synthase F0 subunit 6 (mt-atp6) & -1.3 & 0.739 & 0.999 \\
\hline ATP synthase F0 subunit 8 (mt-atp8) & -1.1 & 0.016 & 0.999 \\
\hline \multicolumn{4}{|l|}{ Glycolysis } \\
\hline Phosphofructokinase, muscle (Pfkm) & 2.1 & $1.00 \mathrm{E}-35$ & $1.00 \mathrm{E}-35$ \\
\hline Pyruvate kinase, muscle (Pkm2) & 2.7 & $1.00 \mathrm{E}-35$ & $1.00 \mathrm{E}-35$ \\
\hline 6-Phosphofructo-2-kinase/fructose-2,6-biphosphatase 3 (Pfkfb3) & 2.9 & 1.19E-13 & $3.26 \mathrm{E}-09$ \\
\hline \multicolumn{4}{|l|}{ Glycogenolysis } \\
\hline Phosphorylase, glycogen, muscle (Pygm) & 1.3 & $1.78 \mathrm{E}-14$ & $4.88 \mathrm{E}-10$ \\
\hline Phosphoglucomutase 1 (Pgm1) & 2.8 & $1.00 \mathrm{E}-35$ & $1.00 \mathrm{E}-35$ \\
\hline Phosphoglucomutase 2-like 1 (Pgm2l1) & 2.2 & 1.19E-13 & $3.25 \mathrm{E}-09$ \\
\hline \multicolumn{4}{|l|}{ Fatty acid ß-oxidation } \\
\hline Carnitine palmitoyltransferase $1 \mathrm{~b}$, muscle (CPT1b) & -1.4 & $3.52 \mathrm{E}-07$ & 0.009 \\
\hline Hydroxyacyl-Coenzyme A dehydrogenase (Hadh) & -1.6 & 2.02E-07 & 0.005 \\
\hline
\end{tabular}


Table 2 Microarray gene expression of soleus muscle-fiber energy metabolism and gene products involved in fibertype regulation (Continued)

\begin{tabular}{lllc}
\hline Regulator genes (muscle fiber-type switch) & & & \\
\hline SRY (sex-determining region Y)-box 6 (Sox6) & 5.2 & $1.00 \mathrm{E}-35$ & $1.00 \mathrm{E}-35$ \\
Myogenic differentiation 1 (Myod1) & 4.4 & $1.11 \mathrm{E}-16$ & $3.06 \mathrm{E}-12$ \\
Myogenin (Myog) & -3.7 & $1.78 \mathrm{E}-09$ & $4.82 \mathrm{E}-05$ \\
Regulator of calcineurin 1 (Rcan1) & -4.6 & $3.51 \mathrm{E}-13$ & $9.61 \mathrm{E}-09$ \\
SIX homeobox 1 (Six1) & 2.0 & $5.68 \mathrm{E}-12$ & $1.55 \mathrm{E}-07$ \\
SIX homeobox 2 (Six2) & 5.0 & $9.39 \mathrm{E}-13$ & $2.57 \mathrm{E}-08$ \\
Myostatin (Mstn) & 4.4 & $3.74 \mathrm{E}-13$ & $1.03 \mathrm{E}-08$ \\
Estrogen-related receptor $\gamma$ (Esrrg) & -2.1 & $1.07 \mathrm{E}-10$ & $2.91 \mathrm{E}-06$ \\
NFAT activating protein with ITAM motif 1 (Nfam1) & -1.7 & $3.34 \mathrm{E}-08$ & $8.96 \mathrm{E}-04$ \\
\hline
\end{tabular}

The fold-change (FC) value indicates the relative amounts of transcripts in rats treated with zidovudine, compared with control animals. The Benjamini Hochberg $q$ value indicates the false-discovery rate.

regulatory factor (MRF) transcribed from myogenin [31]), which is normally expressed predominantly in type I fibers, was downregulated (-3.75-fold; $P=1.78 \mathrm{E}-09$ ) [32].

The homeodomain transcription factors Six 1 and Six2, the transcriptional repressor Sox6, and the transcriptional coactivator Eyal promote a switch from slow to fast fibers [33,34]. We found $\operatorname{Six} 1$ (+2.05-fold; $P=5.68 \mathrm{E}-12)$, Six2 (+5.00-fold; $P=9.39 \mathrm{E}-13)$, Eya 1 ( +1.42 -fold; $P=$ $8.09 \mathrm{E}-06)$, and Sox6 $(+5.20$-fold; $P=1.00 \mathrm{E}-35)$ upregulated in zidovudine-treated rat soleus muscle.

Estrogen-related receptor (ERR) $\gamma$ enhances mitochondrial biogenesis and function, mtDNA content and the expression of contractile proteins specific to slow muscle and is physiologically highly expressed in soleus muscle [35]. We found $E R R \gamma$ downregulated in zidovudineexposed soleus muscle compared with untreated controls (-2.13-fold; $P=1.07 \mathrm{E}-10)$.

Thus, mitochondrial dysfunction is associated with a coordinate regulation of a multitude of transcription factors that orchestrate the transformation from type I to type II fibers.

\section{Discussion}

The present study demonstrates a previously undescribed skeletal muscle fiber-type transformation from slow fibers to fast fibers in a mitochondrial myopathy. The changes in fiber-type composition occur in the absence of muscle regeneration and not only are demonstrated at the level of myosin heavy-chain isoforms and isoforms of other contractile proteins, but also are paralleled by adjustments in the metabolic profile and a switch from an oxidative to a more-glycolytic transcriptosome. From a mechanistic perspective, this response of muscle energetics to the primary defect in respiratory chain function may maintain muscle strength via increased recruitment of glycolysis for ATP production, at the expense of increased energetic cost. The switch to more-glycolytic type II fibers, which are characterized by an increased lactate production compared with type I fibers, could contribute to the hyperlactatemia observed in patients with mitochondriopathies [36]. The fact that hyperlactatemia is typically observed only, or at least is aggravated during exercise in patients with inherited mutations in mtDNA $[37,38]$ can explain the normal lactate levels in our rats in whom blood was collected at rest. Fiber-type switching is also observed in conditions associated with impaired blood oxygenation [39] and diminished muscle microcirculation [40].

In COPD, muscle hypoxia is associated with an increased proportion of type II fibers [39,41], a reduced number of mitochondria [42], increases in glycolytic enzyme activity, and an impairment of oxidative capacity [43].

Patients with idiopathic inflammatory myopathies also reveal an increased proportion of fast fibers and a lower proportion of slow fibers compared with healthy controls [40]. Even in healthy humans exposed to high altitude, the proportion of type I fibers is decreased [44,45]. Although we failed to identify a single master switch of type I to type II fiber transformation, these observations indicate that an impairment of mitochondrial respiration of many causes promotes type II fiber formation.

Interestingly, slow fibers showed even less mtDNA content than did fast fibers in zidovudine-treated rats. This observation may be explained by the dynamics of the system (for example, the possibility that these slow fibers could still be in the process of converting). Alternatively, this finding could be explained with a physiologically higher mtDNA turnover in slow (oxidative) fibers compared with fast (glycolytic) fibers, and therefore an increased susceptibility to the inhibition of mtDNA replication conferred by zidovudine. Clearly, regulators of fiber type exist in addition to mtDNA content, and vice versa. Downregulation of the ERR $\gamma$ may explain some of the biologic processes observed in our model, as ERR $\gamma$ physiologically promotes a switch to slow muscle fibers and induces oxidative metabolism by increasing mitochondrial number, size, and functions [35]. 
Type II fibers in our study had a higher degree of atrophy than did type I fibers, despite the fact that the former appeared to be less dependent on mtDNA replication than the latter, as evidenced by a lesser degree of mtDNA depletion. Because muscle disuse affects mainly type I fiber diameters [46,47], the type II fiber atrophy in our model suggests a mechanism related to mitochondrial dysfunction. This hypothesis is further supported by the predominant type II fiber atrophy in other conditions associated with muscle hypoxia, such as COPD [41], systemic sclerosis [2-4,48], and inflammatory myopathies [5]. Age-related sarcopenia is also associated with a predominant atrophy of type II fibers and an increased abundance of fast myosin heavy-chain isoforms in soleus muscle [49]. It is interesting to speculate, whether mitochondrial dysfunction, which has also been implicated in aging, may be a driver of these characteristics of the aging muscle [50]. Myostatin has been described as a potent negative regulator of muscle mass, and increased myostatin expression is particularly associated with type II atrophy [51]. Consistent with this, we found a fourfold enhancement of myostatin transcription (Table 2). Sarcopenia, in combination with the disabled aerobic energy supply of slow-twitch fibers, can also explain muscle weakness on static and dynamic exercise, fatigue and muscle atrophy observed in patients with mitochondrial myopathies [52].

The effects of mitochondrial dysfunction and hypoxia on fiber-type composition have important clinical implications for training and rehabilitations programs by suggesting that exercise intolerance in mitochondrial dysfunction may be improved not only by cardiopulmonary mechanisms, but also by promoting fiber type II formation, either by resistance training, or pharmacologically by targeting the calcineurin-dependent nuclear factor of activated T-cells (NFAT) with calcineurin inhibitors $[53,54]$.

In mitochondrial myopathies, muscle strength and oxidative capacity were improved without type I fiber enhancement $[55,56]$, and in COPD, muscle strength and oxidative capacity were enhanced without alterations in lung function [41,57]. In idiopathic inflammatory myopathy, however, endurance training increased type I fiber proportions and diameters [40]. This difference could be explained by the preservation of mitochondrial function in idiopathic inflammatory myopathy, which enables type I fiber formation, and the impairment of mitochondrial function in inherited or acquired defects of the mitochondrial genome, which disables type I fiber formation. Clearly, more research is needed about the different effects of training programs on cardiopulmonary function, skeletal muscle microcirculation, oxidative capacity, and fiber-type composition in these different conditions.

\section{Conclusions}

Our work demonstrates a type I to type II fiber transformation in a mitochondrial myopathy and a preferential atrophy in type II fibers. The skeletal muscle fiber-type transformation in the absence of fiber-type regeneration and observed adjustments from oxidative to glycolytic metabolism provide evidence for mitochondrial function as a new regulator of skeletal muscle fiber type and other metabolic capacities. The effects of mitochondrial dysfunction on fiber-type composition have important clinical implications for training and rehabilitations programs.

\section{Abbreviations}

COPD: chronic obstructive pulmonary disease; COX: cytochrome c oxidase; ERR: estrogen-related receptor; IQR: interquartile ranges; MHC: myosin heavy chain; MRF: myogenic regulatory factor; mtDNA: mitochondrial DNA; $\mathrm{NADH}$ $\mathrm{DH}$ : nicotinamide adenine dinucleotide hydrogen dehydrogenase; nDNA: nuclear DNA; SD: standard deviation; SDH: succinate dehydrogenase.

\section{Acknowledgements}

This work was supported by DFG grant VE 492/2-1. The article-processing charge was funded by the German Research Foundation (DFG) and the Albert Ludwigs University Freiburg in the funding program Open Access Publishing. We also thank Karin Sutter and Carmen Kopp for expert technical assistance.

\section{Author details}

${ }^{1}$ Department of Rheumatology \& Clinical Immunology, University Medical Center Freiburg, Hugstetter Str. 55, Freiburg, 79104, Germany. ${ }^{2}$ Department of Hematology \& Oncology, University Medical Center Freiburg, Hugstetter Str. 55, Freiburg, 79104, Germany. ${ }^{3}$ Department of Clinical Chemistry, University Medical Center Freiburg, Hugstetter Str. 55, Freiburg, 79104, Germany. ${ }^{4}$ Department of Neuropediatrics and Muscle Disorders, University Medical Center Freiburg, Hugstetter Str. 55, Freiburg, 79104, Germany. ${ }^{5}$ Department of Rheumatology, Basel University, Burgfelderstr. 101, Basel, 4012, Switzerland.

\section{Authors' contributions}

$\mathrm{DL}$ and ACV carried out the experiments, participated in the design of the study and participated in writing the manuscript. DP performed the microarray analyses. NV and UAW conceived the study, participated in its design and coordination, analyzed the data, and wrote the manuscript. JBK participated in muscle-fiber analyses. EB carried out the blood analyses. All authors read and approved the final manuscript.

\section{Competing interests}

The authors have no competing interests.

Received: 11 May 2012 Revised: 25 September 2012

Accepted: 26 October 2012 Published: 29 October 2012

\section{References}

1. Emslie-Smith AM, Engel AG: Microvascular changes in early and advanced dermatomyositis: a quantitative study. Ann Neurol 1990, 27:343-356.

2. Banker BQ: Dermatomyostis of childhood, ultrastructural alteratious of muscle and intramuscular blood vessels. J Neuropathol Exp Neurol 1975, 34:46-75.

3. Kissel JT, Halterman RK, Rammohan KW, Mendell JR: The relationship of complement-mediated microvasculopathy to the histologic features and clinical duration of disease in dermatomyositis. Arch Neurol 1991, 48:26-30.

4. Fleming JN, Schwartz SM: The pathology of scleroderma vascular disease. Rheum Dis Clin North Am 2008, 34:41-55.

5. Jobin J, Maltais F, Doyon JF, LeBlanc P, Simard PM, Simard AA, Simard C: Chronic obstructive pulmonary disease: capillarity and fiber-type characteristics of skeletal muscle. J Cardiopulmon Rehabil 1998, 18:432-437. 
6. DiMauro S: Mitochondrial myopathies. Curr Opin Rheumatol 2006, 18:636-641.

7. DiMauro S, Schon EA: Mitochondrial respiratory-chain diseases. N Engl J Med 2003, 348:2656-2668.

8. Sirvent P, Fabre O, Bordenave S, Hillaire-Buys D, Raynaud De ME, Lacampagne A, Mercier J: Muscle mitochondrial metabolism and calcium signaling impairment in patients treated with statins. Toxicol Appl Pharmacol 2012, 259:263-268.

9. Lebrecht D, Deveaud C, Beauvoit B, Bonnet J, Kirschner J, Walker UA: Uridine supplementation antagonizes zidovudine-induced mitochondrial myopathy and hyperlactatemia in mice. Arthritis Rheum 2008, 58:318-326.

10. Owczarek J, Jasinska M, Orszulak-Michalak D: Drug-induced myopathies: an overview of the possible mechanisms. Pharmacol Rep 2005, 57:23-34.

11. Caiozzo VJ, Baker MJ, Huang K, Chou H, Wu YZ, Baldwin KM: Single-fiber myosin heavy chain polymorphism: how many patterns and what proportions? Am J Physiol Regul Integr Comp Physiol 2003, 285:R570-R580.

12. Termin A, Staron RS, Pette D: Changes in myosin heavy chain isoforms during chronic low-frequency stimulation of rat fast hindlimb muscles: a single-fiber study. Eur J Biochem 1989, 186:749-754.

13. Izumo S, Nadal-Ginard B, Mahdavi V: All members of the MHC multigene family respond to thyroid hormone in a highly tissue-specific manner. Science 1986, 231:597-600.

14. Lewis W, Day BJ, Copeland WC: Mitochondrial toxicity of NRTI antiviral drugs: an integrated cellular perspective. Nat Rev Drug Discov 2003, 2:812-822.

15. Arnaudo E, Dalakas M, Shanske S, Moraes CT, DiMauro S, Schon EA: Depletion of muscle mitochondrial DNA in AIDS patients with zidovudine-induced myopathy. Lancet 1991, 337:508-510.

16. Collins ML, Sondel N, Cesar D, Hellerstein MK: Effect of nucleoside reverse transcriptase inhibitors on mitochondrial DNA synthesis in rats and humans. J Acquir Immune Defic Syndr 2004, 37:1132-1139.

17. Deveaud C, Beauvoit B, Hagry S, Galinier A, Carriere A, Salin B, Schaeffer J, Caspar-Bauguil S, Fernandez Y, Gordien JB, Breilh D, Penicaud L, Casteilla L, Rigoulet M: Site specific alterations of adipose tissue mitochondria in $3^{\prime}-$ azido-3'-deoxythymidine (AZT)-treated rats: an early stage in lipodystrophy? Biochem Pharmacol 2005, 70:90-101.

18. Nau R, Schunck O: Cannulation of the lateral saphenous vein: a rapid method to gain access to the venous circulation in anaesthetized guinea pigs. Lab Anim 1993, 27:23-25.

19. U.S. Department of Health \& Human Services, Office of laboratory animal welfare. [http://grants.nih.gov/grants/olaw/olaw.htm].

20. Round JM, Matthews Y, Jones DA: A quick, simple and reliable histochemical method for ATPase in human muscle preparations. Histochem J 1980, 12:707-710.

21. Sciacco M, Bonilla E: Cytochemistry and immunocytochemistry of mitochondria in tissue sections. Methods Enzymol 1996, 264:509-521.

22. Lebrecht D, Setzer B, Ketelsen UP, Haberstroh J, Walker UA: Timedependent and tissue-specific accumulation of mtDNA and respiratory chain defects in chronic doxorubicin cardiomyopathy. Circulation 2003, 108:2423-2429

23. DiMauro S, Mendell JR, Sahenk Z, Bachman D, Scarpa A, Scofield RM, Reiner C: Fatal infantile mitochondrial myopathy and renal dysfunction due to cytochrome-c-oxidase deficiency. Neurology 1980, 30:795-804

24. Sciacco M, Bonilla E, Schon EA, DiMauro S, Moraes CT: Distribution of wildtype and common deletion forms of mtDNA in normal and respirationdeficient muscle fibers from patients with mitochondrial myopathy. Hum Mol Genet 1994, 3:13-19.

25. Lebrecht D, Vargas Infante YA, Setzer B, Kirschner J, Walker UA: Uridine supplementation antagonizes zalcitabine-induced microvesicular steatohepatitis in mice. Hepatology 2007, 15:72-79.

26. Irizarry RA, Hobbs B, Collin F, Beazer-Barclay YD, Antonellis KJ, Scherf U, Speed TP: Exploration, normalization, and summaries of high density oligonucleotide array probe level data. Biostatistics 2003, 4:249-264.

27. EMBL-EBI, ArrayExpress. [http://ebi.ac.uk/arrayexpress].

28. Benjamini $Y$, Hochberg $Y$ : Controlling the false discovery rate: a practical and powerful approach to multiple testing. I R Stat Soc 1995, , B57: 289-300.

29. Berg JS, Powell BC, Cheney RE: A millennial myosin census. Mol Biol Cell 2001, 12:780-794

30. Hughes SM, Taylor JM, Tapscott SJ, Gurley CM, Carter WJ, Peterson CA Selective accumulation of MyoD and myogenin mRNAs in fast and slow adult skeletal muscle is controlled by innervation and hormones. Development 1993, 118:1137-1147.
31. Hughes SM, Chi MM, Lowry OH, Gundersen K: Myogenin induces a shift of enzyme activity from glycolytic to oxidative metabolism in muscles of transgenic mice. J Cell Biol 1999, 145:633-642.

32. Voytik SL, Przyborski M, Badylak SF, Konieczny SF: Differential expression of muscle regulatory factor genes in normal and denervated adult rat hindlimb muscles. Dev Dyn 1993, 198:214-224.

33. Grifone R, Laclef C, Spitz F, Lopez S, Demignon J, Guidotti JE, Kawakami K, Xu PX, Kelly R, Petrof BJ, Daegelen D, Concordet JP, Maire P: Six1 and Eya1 expression can reprogram adult muscle from the slow-twitch phenotype into the fast-twitch phenotype. Mol Cell Biol 2004, 24:6253-6267.

34. Hagiwara N, Ma B, Ly A: Slow and fast fiber isoform gene expression is systematically altered in skeletal muscle of the Sox6 mutant, $\mathrm{p} 100 \mathrm{H}$. Dev Dyn 2005, 234:301-311.

35. Rangwala SM, Wang X, Calvo JA, Lindsley L, Zhang Y, Deyneko G, Beaulieu V, Gao J, Turner G, Markovits J: Estrogen-related receptor gamma is a key regulator of muscle mitochondrial activity and oxidative capacity. J Biol Chem 2010, 285:22619-22629.

36. Chariot P, Monnet I, Mouchet M, Rohr M, Lefaucheur J-P, DubreuilLemaire ML, Chousterman M, Gherardi R: Determination of the blood lactate:pyruvate ratio as a noninvasive test for the diagnosis of zidovudine myopathy. Arthritis Rheum 1994, 37:583-586.

37. Mancuso M, Orsucci D, LoGerfo A, Rocchi A, Petrozzi L, Nesti C, Galetta F, Santoro G, Murri L, Siciliano G: Oxidative stress biomarkers in mitochondrial myopathies, basally and after cysteine donor supplementation. J Neurol 2010, 257:774-781.

38. Munnich A, Rotig A, Chretien D, Saudubray JM, Cormier V, Rustin P: Clinical presentations and laboratory investigations in respiratory chain deficiency. Eur J Pediatr 1996, 155:262-274.

39. Hildebrand IL, Sylven C, Esbjornsson M, Hellstrom K, Jansson E: Does chronic hypoxaemia induce transformations of fibre types? Acta Physiol Scand 1991, 141:435-439.

40. Dastmalchi M, Alexanderson H, Loell I, Stahlberg M, Borg K, Lundberg IE, Esbjornsson M: Effect of physical training on the proportion of slowtwitch type I muscle fibers, a novel nonimmune-mediated mechanism for muscle impairment in polymyositis or dermatomyositis. Arthritis Rheum 2007, 57:1303-1310

41. Rabinovich RA, Vilaro J: Structural and functional changes of peripheral muscles in chronic obstructive pulmonary disease patients. Curr Opin Pulmon Med 2010, 16:123-133.

42. Gosker HR, Hesselink MK, Duimel H, Ward KA, Schols AM: Reduced mitochondrial density in the vastus lateralis muscle of patients with COPD. Eur Respir J 2007, 30:73-79.

43. Howald H, Pette D, Simoneau JA, Uber A, Hoppeler H, Cerretelli P: Effect of chronic hypoxia on muscle enzyme activities. Int I Sports Med 1990, 11(Suppl 1):S10-S14.

44. Green HJ, Sutton JR, Cymerman A, Young PM, Houston CS: Operation Everest II: adaptations in human skeletal muscle. J App/ Physiol 1989, 66:2454-2461.

45. Bigard AX, Brunet A, Guezennec CY, Monod H: Skeletal muscle changes after endurance training at high altitude. J Appl Physiol 1991, 71:2114-2121.

46. Zhong H, Roy RR, Siengthai B, Edgerton VR: Effects of inactivity on fiber size and myonuclear number in rat soleus muscle. J Appl Physiol 2005, 99:1494-1499.

47. Booth FW, Gollnick PD: Effects of disuse on the structure and function of skeletal muscle. Med Sci Sports Exerc 1983, 15:415-420.

48. Scarpelli M, Montironi R, Tulli D, Sisti S, Matera G, Magi GC, Collan Y Quantitative analysis of quadriceps muscle biopsy in systemic sclerosis. Pathol Res Pract 1992, 188:603-606.

49. Carter EE, Thomas MM, Murynka T, Rowan SL, Wright KJ, Huba E, Hepple RT: Slow twitch soleus muscle is not protected from sarcopenia in senescent rats. Exp Gerontol 2010, 45:662-670.

50. Trounce I, Byrne E, Marzuki S: Decline in skeletal muscle mitochondrial respiratory chain function: possible factor in aging. Lancet 1989, i:637-639.

51. Jespersen JG, Nedergaard A, Andersen $L L$, Schjerling P, Andersen JL: Myostatin expression during human muscle hypertrophy and subsequent atrophy: increased myostatin with detraining. Scand J Med Sci Sports 2011, 21:215-223.

52. Dalakas MC, Illa I, Pezeshkpour GH, Laukaitis JP, Cohen B, Griffin JL: Mitochondrial myopathy caused by long-term zidovudine therapy. N Engl J Med 1990, 322:1098-1105. 
53. Chin ER, Olson EN, Richardson JA, Yang Q, Humphries C, Shelton JM, Wu H, Zhu W, Bassel-Duby R, Williams RS: A calcineurin-dependent transcriptional pathway controls skeletal muscle fiber type. Genes Dev $1998,12: 2499-2509$.

54. Schiaffino $\mathrm{S}$, Reggiani C: Fiber types in mammalian skeletal muscles. Physiol Rev 2011, 91:1447-1531.

55. Murphy JL, Blakely EL, Schaefer AM, He L, Wyrick P, Haller RG, Taylor RW Turnbull DM, Taivassalo T: Resistance training in patients with single, large-scale deletions of mitochondrial DNA. Brain 2008, 131:2832-2840.

56. Jeppesen TD, Schwartz M, Olsen DB, Wibrand F, Krag T, Duno M, Hauerslev S, Vissing J: Aerobic training is safe and improves exercise capacity in patients with mitochondrial myopathy. Brain 2006 129:3402-3412

57. Vogiatzis I, Terzis G, Nanas S, Stratakos G, Simoes DC, Georgiadou O, Zakynthinos S, Roussos C: Skeletal muscle adaptations to interval training in patients with advanced COPD. Chest 2005, 128:3838-3845.

doi:10.1186/ar4076

Cite this article as: Venhoff et al:: Muscle-fiber transdifferentiation in an experimental model of respiratory chain myopathy. Arthritis Research \& Therapy 2012 14:R233.

\section{Submit your next manuscript to BioMed Central} and take full advantage of:

- Convenient online submission

- Thorough peer review

- No space constraints or color figure charges

- Immediate publication on acceptance

- Inclusion in PubMed, CAS, Scopus and Google Scholar

- Research which is freely available for redistribution

Submit your manuscript at www.biomedcentral.com/submit 